\title{
DmCatD, A CATHEPSIN D-LIKE PEPTIDASE OF THE HEMATOPHAGOUS INSECT DIPETALOGASTER MAXIMA (HEMIPTERA: REDUVIIDAE): PURIFICATION, BIOINFORMATIC ANALYSES AND THE SIGNIFICANCE OF ITS INTERACTION WITH LIPOPHORIN IN THE INTERNALIZATION BY DEVELOPING OOCYTES
}

\author{
Jimena Leyriaa ${ }^{a}$ Leonardo L. Fruttero ${ }^{a, b}$, Rodrigo Ligabue-Braun $^{c}$, Marina S. Defferrari $^{d}$, \\ Estela L. Arrese $^{\mathrm{e}}$, José L. Soulages ${ }^{\mathrm{e}}$, Beatriz P. Settembrini ${ }^{f}$, Celia R. Carlini ${ }^{\mathrm{b}, \mathrm{c}}$, and Lilián E. \\ Canavoso $^{\mathrm{a},{ }^{*}}$ \\ aDepartamento de Bioquímica Clínica-CIBICI-CONICET, Facultad de Ciencias Químicas, \\ Universidad Nacional de Córdoba, Córdoba, Argentina \\ bBrain Institute (Instituto do Cérebro-INSCER), Pontifícia Universidade Católica do Rio Grande do \\ Sul, Porto Alegre, Brazil \\ 'Center of Biotechnology, Universidade Federal do Rio Grande do Sul Porto Alegre, RS, Brazil \\ dDepartment of Biology, University of Toronto Mississauga, Mississauga, ON, Canada \\ eDepartment of Biochemistry and Molecular Biology, Oklahoma State University, Stillwater, OK, \\ USA
}

fMuseo Argentino de Ciencias Naturales, Ciudad Autónoma de Buenos Aires, Argentina

\begin{abstract}
DmCatD, a cathepsin D-like peptidase of the hematophagous insect Dipetalogaster maxima, is synthesized by the fat body and the ovary and functions as yolk protein precursor. Functionally, DmCatD is involved in vitellin proteolysis. In this work, we purified and sequenced DmCatD, performed bioinformatic analyses and investigated the events involved in its targeting and storage in developing oocytes. By ion exchange and gel filtration chromatography, DmCatD was purified from egg homogenates and its identity was confirmed by mass spectrometry. Approximately $73 \%$ of the full-length transcript was sequenced. The phylogeny indicated that DmCatD has features which suggest its distancing from "classical" cathepsins D. Bioinformatic analyses using a chimeric construct were employed to predict post-translational modifications. Structural modeling showed that DmCatD exhibited the expected folding for this type of enzyme, and an active site with conserved architecture. The interaction between DmCatD and lipophorin in the hemolymph was demonstrated by co-immunoprecipitation. Colocalization of both proteins in developing oocyte membranes and yolk bodies was detected by immunofluorescence. Docking assays favoring the interaction DmCatD-lipophorin were carried out after modeling lipophorin of a
\end{abstract}

\footnotetext{
*Corresponding author: lcanavo@fcq.unc.edu.ar (LEC). Tel. + 54351 535-3850; Fax: + 54 351 433-3048.
} 
related triatomine species. Our results suggest that lipophorin acts as a carrier for DmCatD to facilitate its further internalization by the oocytes. The mechanisms involved in the uptake of peptidases within the oocytes of insects have not been reported. This is the first experimental work supporting the interaction between cathepsin $\mathrm{D}$ and lipophorin in an insect species, enabling us to propose a pathway for its targeting and storage in developing oocytes.

\section{Keywords}

Triatominae; cathepsin D; lipophorin; vitellogenesis; oocyte

\section{Introduction}

Triatomines (Hemiptera: Reduviidae) or "kissing bugs" are obligate hematophagous insects with relevance in public health since they are vectors of the protozoan Trypanosoma cruzi, the etiological agent of Chagas' disease. Currently, about 6 to 7 million people worldwide are estimated as infected with the parasite (WHO, 2017), with the highest prevalence in Latin America (Hotez et al., 2008). Because T. cruzi is mainly transmitted to people by triatomines (Miles, 2017), vector control represents the best way to reduce the incidence of the illness.

In insects, vitellogenesis is one of the most important events in reproduction (Raikhel, 2005) and, in triatomine females, it is strongly coupled to the intake of a blood meal (Stoka et al., 1987). Vitellogenesis is characterized by a rapid growth of oocytes due to a remarkable uptake and deposition of proteins, lipids and other molecules. During this process, large amounts of yolk protein precursors synthesized in the fat body and/or in the follicular epithelial cells (Izumi et al., 1994; Melo et al., 2000; Atella et al., 2005) are stored in developing oocytes. Among them, vitellogenin constitutes the main yolk protein precursor that is taken up by the oocytes by receptor-mediated endocytosis and stored as vitellin in specialized lysosomal compartments or yolk bodies (Raikhel and Dhadialla, 1992; Tufail and Takeda, 2008; 2009).

On the other hand, lipophorin is the major insect lipoprotein that carries several lipid classes in the hemolymph to the target tissues (Canavoso et al., 2001). In addition to this physiological role, there are reports indicating the ability of lipophorin to bind other molecules, such as juvenile hormone (Engelmann and Mala, 2000; Zalewska et al., 2009), proteins from the immune system, (Ma et al., 2006; Rahman et al., 2006) and morphogens (Eugster et al., 2007). In several insect species, including triatomines, it was demonstrated that lipophorin can be endocytosed by the oocytes and stored in yolk bodies with vitellin, thus functioning as a yolk protein precursor during vitellogenesis (Kawooya and Law, 1988; Ziegler and Van Antwerpen, 2006; Fruttero et al., 2011; Leyria et al., 2014).

Fertilization triggers embryogenesis, a process in which the yolk proteins are used as substrates for the growing embryo (Yamahama et al., 2005). Different peptidases and acid phosphatases have been associated with the degradation of yolk proteins during embryogenesis (Nussenzveig et al., 1992; Yamamoto and Takahashi, 1993; Izumi et al., 1994; Fialho et al., 2005; Oliveira et al., 2008). Most peptidases are yolk protein precursors 
synthesized in the fat body as well as in the ovary, released subsequently to the hemolymph as pro-enzymes and stored in the oocyte associated with the yolk bodies (Giorgi and Nordin, 2005). It was demonstrated that an acid phosphatase and cathepsin D are important regulators of yolk protein degradation during the embryogenesis of the triatomine Rhodnius prolixus (Fialho et al., 2005; Gomes et al., 2010).

Dipetalogaster maxima is a triatomine species used as a model to assess biochemical, cellular and molecular events during vitellogenic and post-vitellogenic reproduction stages (Aguirre et al., 2008; 2011). Employing this insect we have demonstrated that DmCatD, a cathepsin D-like peptidase, is synthesized by the fat body and the ovary as a yolk protein precursor and stored as an inactive enzyme (pro-DmCatD) in yolk bodies (Leyria et al., 2015). It was also demonstrated that in females of $D$. maxima, blood deprivation promotes follicular atresia and oosorption of terminal oocytes. Such processes were characterized by an early activation of DmCatD, which seems to be part of the mechanisms regulating yolk protein degradation during this post-vitellogenic stage (Leyria et al., 2015). In spite of the physiological relevance of DmCatD in the biology of reproduction in triatomines, the mechanism involving its targeting to the oocyte membrane and posterior internalization during vitellogenesis has not been established. In this work, we have purified a DmCatD peptidase from eggs of D. maxima, reported its transcript sequence and structural properties. In addition, bioinformatic and biochemical approaches were performed to assess the interaction of DmCatD-lipophorin and analyzed the relevance of such potential association for DmCatD internalization in developing oocytes.

\section{Materials and Methods}

\subsection{Ethics statement}

Housing conditions and manipulation of hens employed in the maintenance of the insect colony followed the protocol authorized by the Animal Care Committee of the Centro de Investigaciones en Bioquímica Clínica e Inmunología (CIBICI-CONICET-Universidad Nacional de Córdoba) in accordance with the guidelines published by the Canadian Council on Animal Care with the assurance number A5802-01 delivered by the Office of Laboratory Animal Welfare (National Institutes of Health). The animal facility at the CIBICI-CONICET is a dependency of the Argentine National Ministry of Science (Sistema Nacional de Bioterios, MINCyT, http://www.bioterios.mincyt.gob.ar). No infective insect species, human blood or hen sacrifice were involved in the study. Details of the approved protocol were recently published (Leyria et al., 2015).

\subsection{Chemicals}

Rabbit polyclonal anti-cathepsin D (catalog code sc-10725) and rabbit anti-ATP5B/ $\beta$-chain of ATP synthase ( $\beta$-ATPase, catalog code sc-33618) antibodies, both of human origin, were from Santa Cruz Biotechnology (Palo Alto, CA, USA). The cross-reactivity between the anti-cathepsin D antibody and DmCatD was already reported (Leyria et al., 2015). Goat antirabbit IgG labeled with Alexa Fluor ${ }^{\circledR} 568$ antibody (Molecular Probes, Eugene, OR, USA); Tissue-Tek embedding medium Optimal Cutting Temperature (OCT) (Miles, Elkhart, IN, USA); MMLV reverse transcriptase (Promega, Heidelberg, Germany); Platinum® Taq DNA 
Polymerase High Fidelity (Thermo Fisher Scientific, Waltham, MA, USA); primers (Sigma Genosys, Houston, TX, USA); MasterPure RNA Purification Kit (Epicenter Biotechnologies, Madison, WI, USA); Fluorsave (Calbiochem, Darmstadt, Germany) and Color Prestained Protein Standard (New England Biolabs Inc., Ipswich, MA, USA) were from the indicated commercial sources. The fluorogenic peptide substrate Abz-AIAFFSRQEDDnp (Abz, orthoaminobenzoic acid, EDDnp, ethylenediamine-2,4-dinitrophenyl) was a kind gift from Dr. Maria Aparecida Juliano (Universidade Federal de São Paulo, Brazil). Bovine serum albumin (BSA), dimethylpimelimidate (DMP), fetal bovine serum (FBS), anti-mouse IgG conjugated to FITC antibody and all other chemicals were from SigmaAldrich (St. Louis, MO, USA).

\subsection{Insects}

Experiments were carried out with insects taken from a colony of $D$. maxima, maintained at $28{ }^{\circ} \mathrm{C}, 70 \%$ relative humidity, $8: 16 \mathrm{~h}$ light:dark photoperiod. Insects were fed on hen blood (Canavoso and Rubiolo, 1995), according to the recommendations of the National Institute of Parasitology (Health Ministry, Argentina) (Nuñez and Segura, 1987). Standardized conditions of insect rearing were previously described (Aguirre et al., 2008). Briefly, fifthinstar females were separated from the males before feeding. Newly emerged females were segregated individually and placed together with two recently fed males during $48 \mathrm{~h}$. Mating was checked by observation of the spermatophore. Mated females were kept in individual containers until they were able to feed a blood meal (days 10-12 post-ecdysis), which resulted in a 3.0-5.5-fold increase in the body weight of the insect. Experimental approaches were performed using vitellogenic females at days 4-6 after blood feeding (Aguirre et al., 2008; Leyria et al., 2015).

\subsection{Purification of DmCatD from eggs}

In a typical purification experiment, 60 eggs from $D$. maxima collected within 24 h post oviposition were homogenized using a Potter-Elvehjem (15 strokes) in cold $20 \mathrm{mM}$ sodium phosphate buffer (NaPB), pH 6.0 with the addition of protease inhibitors E64 and PMSF at final concentrations of 15 and $80 \mu \mathrm{M}$, respectively. The homogenate was centrifuged twice at 5,000 x $g\left(10 \mathrm{~min}\right.$ each, $\left.4{ }^{\circ} \mathrm{C}\right)$ and the supernatant was recovered to proceed with the protein determination (Bradford, 1976).

Fractions from purification steps were analyzed using the fluorogenic substrate for cathepsin D, Abz-AIAFFSRQ-EDDnp, as reported previously (Aguirre et al., 2011; Leyria et al., 2015). Additional activity assays were conducted in presence of the aspartyl protease inhibitor pepstatin A.

2.4.1. Cation-exchange chromatography-The first purification step of the egg homogenate was performed by Fast Protein Liquid Chromatography (FPLC), using a column packed with $10 \mathrm{ml}$ of CM-Sepharose Fast-Flow resin (GE Healthcare, Amersham Biosciences, Little Chalfont, England) which was equilibrated with $20 \mathrm{mM} \mathrm{NaPB}$, pH 6.0. The egg homogenate was loaded and the column was washed with buffer to remove nonretained proteins, then, bound proteins were eluted with $\mathrm{NaPB}$ at increasing concentrations of $\mathrm{NaCl}(100,200,300$ and $500 \mathrm{mM})$. Non-retained and eluted fractions were collected and 
assayed for cathepsin D activity as described previously (Aguirre et al., 2011). The fraction eluted with $200 \mathrm{mM} \mathrm{NaCl}$ showed the highest enzymatic activity and was subsequently concentrated using a $10 \mathrm{kDa}$ cut off Centriprep ${ }^{\mathrm{TM}}$ Centrifugal Filter Concentrators (EMD Millipore, Billerica, MA, USA).

2.4.2. Size exclusion chromatography-Gel filtration was performed using a Superdex 75 column $(1.2 \times 30 \mathrm{~cm})(\mathrm{GE}$ Healthcare, Amersham Biosciences, Little Chalfont, England) equilibrated in $20 \mathrm{mM} \mathrm{NaPB} \mathrm{pH} \mathrm{6.0.} \mathrm{Two} \mathrm{milliliters} \mathrm{of} \mathrm{the} \mathrm{sample} \mathrm{eluted} \mathrm{from} \mathrm{the}$ cation-exchange chromatography column at $200 \mathrm{mM} \mathrm{NaCl}$ were applied to the column and protein peaks, monitored at $280 \mathrm{~nm}$, were individually collected in $0.5 \mathrm{ml}$ fractions and assayed for enzymatic activity.

\subsection{Electrophoresis, in-gel trypsin digestion and mass spectrometry analysis}

Sodium dodecyl sulfate-polyacrylamide gel electrophoresis (SDS-PAGE) was performed as described by Laemmli (1970) in a $12 \%$ separating gel to visualize the purified protein.

Protein bands were manually excised from Coomassie stained gels and digested in-gel with trypsin. The preparation for mass spectrometry was performed as reported in Fruttero et al. (2014) with minor modifications. To identify proteins, a liquid chromatography (LC) separation (reversed-phase High Performance Liquid Chromatography, HPLC) coupled with tandem mass spectrometry (MS/MS) strategy was used. MS/MS analyses were performed in an electrospray ionization (ESI)-Q-Exactive mass spectrometer equipped with a High Collision Dissociation cell and an Orbitrap analyzer coupled to an EASY-nLC 1000 Liquid Chromatography system (Thermo Scientific, Waltham, MA, USA) at the CEQUIBIEM mass spectrometry facility (Centro de Estudios Químicos y Biológicos por Espectrometría de Masas, FCEyN, UBA, Argentina). The MS/MS spectra were analyzed using Proteome discoverer v. 1.4 software (Thermo Scientific, Waltham, MA, USA) and the D. maxima (AHE57676) and T. infestans (AEO94539) databases of The National Center for Biotechnology Information (NCBI) were employed for the analyses. Search parameters allowed a maximum of two missed cleavages, the carbamidomethylation of cysteine, the possible oxidation of methionine, precursor mass tolerance of $10 \mathrm{ppm}$ and fragment mass tolerance of 0.05 Da. Only the matches considered of high confidence by the software were taken into account.

\subsection{RT-PCR and sequencing}

In order to obtain the RNA sequence of DmCatD, Reverse Transcription - Polymerase chain reaction (RT-PCR) experiments were performed. For RNA extraction, dissected fat bodies of three females were pooled and the MasterPure RNA Purification Kit was used according to the manufacturer's protocol. To eliminate genomic DNA, samples were treated with DNAse provided in the kit. RNA integrity was evaluated by electrophoresis in a $1 \%$ agarose gel, and only the band corresponding to $18 \mathrm{~S}$ rRNA was observed. It is important to highlight that $28 \mathrm{~S}$ rRNA of most insects contains an endogenous "hidden break" upon denaturation, the masking hydrogen bonds are disrupted, releasing two similar sized fragments that both migrate closely with 18S rRNA (Winnebeck et al., 2010). cDNA was synthesized from $2 \mu \mathrm{g}$ of total RNA by reverse transcription reaction using oligo dT and the MMLV reverse 
transcriptase protocol. For PCR, Platinum ${ }^{\circledR}$ Taq DNA Polymerase High Fidelity was used. Since the genome of $D$. maxima is not available, the design of primers was based on the mRNA sequence of the Triatoma infestans cathepsin D (ID JN606068.1). Primers were: 5' GCTCTTCAAATCTTTGGATACCATC-3' (sense) and 5' TTATTGTTTCAAACTGGCAAAGCTAAC- $3^{\prime}$ (antisense). Using this set of primers we were able to obtain a product of approximately $902 \mathrm{bp}$, encompassing about $73 \%$ of the full-length sequence of the transcript (lacking the N-terminus). PCR reactions were carried out and the products were processed using the sequencing service of the Department of Biochemistry and Molecular Biology, Oklahoma State University, OK, USA.

\subsection{Bioinformatic analyses for DmCatD}

The deduced amino acid sequence, molecular mass and isoelectric point prediction were assessed using tools available on ExPASy (www.expasy.org - SIB Bioinformatics Resource Portal) (Artimo et al., 2012). The active site of the peptidase was detected by ScanProsite (Sigrist et al., 2013). The bioinformatic tool from NCBI was used to analyze highly conserved regions of DmCatD. The prediction of post-translational modifications was carried out with CBS (http://www.cbs.dtu.dk/services/) and ScanProsite (http:// prosite.expasy.org/scanprosite/), through multiple tools (Blom et al., 1999; Petersen et al., 2011; Sigrist et al., 2013). Modifications examined included the occurrence of phosphorylation and N-myristoylation sites. The DmCatD from D. maxima structural model was built based on crystallographic data from proteins with similar secondary structure arrangements, using Phyre2 server (Kelley et al., 2015). The structure was stereochemically evaluated using Procheck (Laskowski et al., 1993). The R. prolixus lipophorin structural modeling (corresponding to the apolipophorin II/I gene, VectorBase ID RPRC002125-PA) was carried out with I-TASSER (Zhang, 2008; Roy et al., 2010; Yang et al., 2015), while the DmCatD-lipophorin docking simulation was performed with two independent macromolecular docking programs with no positional biases: PatchDock (SchneidmanDuhovny et al., 2005) and PIPER (Kozakov et al., 2006) via ClusPro 2.0 (Comeau et al., 2004).

Hydrophobicity profiles were calculated with the Kyte-Doolittle scale (Kyte and Doolittle, 1982), and the electrostatic surfaces were generated with APBS tools (Baker et al., 2001) under UCSF Chimera (Pettersen et al., 2004).

Evolutionary analyses were conducted with MEGA6 (Tamura et al., 2013). The phylogenetic tree for DmCatD was inferred using the Maximum Likelihood method (LG+G) (Le and Gascuel, 2008). Confidence was assessed by bootstrap pseudo-replications (1000 rounds). Sequences with similarity to DmCatD were searched with BlastP (Johnson et al., 2008) and employed in the tree reconstruction.

Except for the evolutionary analysis, all bioinformatics studies were conducted with a chimeric construct that included the $\mathrm{N}$-terminus region of T. infestans cathepsin D.

\subsection{Hemolymph collection}

The hemolymph was collected with a Hamilton syringe from immobilized vitellogenic females at days 4-6 after blood feeding. Their legs were sectioned at the level of the coxa 
and the hemolymph was collected into cold microtubes, in the presence of $10 \mathrm{mM}$ $\mathrm{Na}_{2}$ EDTA, $5 \mathrm{mM}$ dithiothreitol and a cocktail of protease inhibitors: $1 \mathrm{mM}$ phenylmethylsulfonyl fluoride (PMSF), $1 \mathrm{mM} \mathrm{N}$-a-p-tosyl-L-lysine chloromethyl ketone (TLCK), $1 \mathrm{mM}$ pepstatin A and $0.3 \mathrm{mM}$ aprotinin (Fruttero et al., 2009). Samples were centrifuged at $10,000 \times \mathrm{g}$ for $5 \mathrm{~min}$ at $4{ }^{\circ} \mathrm{C}$ to remove hemocytes and then stored at $-70{ }^{\circ} \mathrm{C}$, after protein determination (Bradford, 1976).

\subsection{Co-immunoprecipitation of DmCatD with lipophorin}

Co-immunoprecipitation assays using hemolymph from vitellogenic females were performed as described previously (Fruttero et al., 2017). Anti- $\beta$-ATPase (control) and anticathepsin D antibodies ( $0.2 \mu \mathrm{g}$ each) were covalently coupled to protein A Mag Sepharose beads (GE Healthcare, Little Chalfont, UK) by their incubation for $1 \mathrm{~h}$ at room temperature. The magnetic beads were washed with a 5-fold dilution of the sample in Tris buffered saline (TBS, $50 \mathrm{mM}$ Tris, $150 \mathrm{mM} \mathrm{NaCl}, \mathrm{pH}$ 7.5) and further recovered with a magnetic rack. After two washes, the beads were incubated with $200 \mathrm{mM}$ triethanolamine buffer ( $\mathrm{pH} 8.9$ ) containing $50 \mathrm{mM}$ DMP for $1 \mathrm{~h}$ at room temperature. The beads were washed with triethanolamine buffer as already stated and blocked with $100 \mathrm{mM}$ ethanolamine buffer $(\mathrm{pH}$ 8.9) for $15 \mathrm{~min}$ at room temperature. The elution buffer ( $0.1 \mathrm{M}$ glycine, $2 \mathrm{M}$ urea, $\mathrm{pH}$ 2.9) was added to remove the unbound antibody and then beads were washed three times with TBS. Hemolymph or rat brain homogenates (control), both containing $60 \mu \mathrm{g}$ of total proteins were incubated with the antibodies covalently coupled to protein A Mag Sepharose beads (anti- $\beta$-ATPase or anti-cathepsin D antibodies, $1 \mathrm{~h}$ at room temperature, with slow end-overend mixing). The proteins bound to the beads were eluted with the elution buffer and protein A Mag Sepharose beads were removed using a magnetic rack. The eluted proteins, the input and the standard were subjected to Tris-Tricine-SDS gel electrophoresis as described elsewhere (Fruttero et al., 2014). The immunodetection of DmCatD-lipophorin interaction was performed by western blot, using an anti-lipophorin antibody (anti-Lp, 1:1,000) obtained as described previously (Canavoso and Rubiolo, 1995). The secondary antibody, Li-Cor IRDye $800 \mathrm{CW}$ polyclonal goat anti-rabbit $\operatorname{IgG}(1: 15,000)$ was incubated at room temperature for $1 \mathrm{~h}$. Both antibodies were diluted in TBS- $0.1 \%$ Tween 20 containing $5 \%$ non-fat milk. After washing, blots were scanned and analyzed with the Odyssey quantitative western blot near-infrared system (Li-Cor Biosciences, Lincoln, NE, USA) using default settings. DmCatD-vitellogenin interaction in the hemolymph of vitellogenic females was also tested employing a similar protocol but using an anti-vitellin antibody (anti-Vt, 1:1,000) obtained as described previously (Aguirre et al. 2008).

\subsection{Colocalization of DmCatD with lipophorin in the ovarian tissue}

Ovaries from vitellogenic females at days 4-6 after blood feeding were dissected out in cold phosphate buffered saline (PBS, $6.6 \mathrm{mM} \mathrm{Na} 2 \mathrm{HPO}_{4} / \mathrm{KH}_{2} \mathrm{PO}_{4}, 150 \mathrm{mM} \mathrm{NaCl}$, $\mathrm{pH}$ 7.4), using a standard stereoscope with an optic fiber light source and processed for cryostat sectioning as reported previously (Leyria et al., 2015). Tissue sections of $8 \mu \mathrm{m}$ were obtained with a Leica CM1510 cryostat (Leica Microsystems, Wetzlar, Germany) and placed onto poly-Llysine-treated glass slides. Ovarian sections were incubated with $1 \%$ BSA and $5 \%$ FBS in PBS to block non-specific binding sites. The slides were sequentially incubated with the anti-cathepsin D (1:100), the anti-rabbit IgG labeled with Alexa Fluor® 568 (1:400) and the 
anti-Lp conjugated to fluorescein isothiocyanate (FITC, 1:40) antibodies. Antibodies were diluted in $1 \%$ BSA in PBS. All incubations were performed inside a humid chamber at $37^{\circ} \mathrm{C}$ for $1 \mathrm{~h}$. Slides were rinsed twice with PBS for $5 \mathrm{~min}$. Control experiments were carried out by omitting one or a combination of the following antibodies: anti-rabbit IgG coupled to Alexa Fluor ${ }^{\circledR}$ 568, anti-cathepsin D or anti-Lp-FITC. An additional control for autofluorescence was conducted using an anti-mouse IgG antibody conjugated to FITC (irrelevant antibody, 1:40). Slides were rinsed with PBS, air-dried, mounted in Fluorsave and observed with an Olympus FV300 laser scanning confocal microscope (Olympus, Tokyo, Japan) equipped with 488 and $543 \mathrm{~nm}$ lasers. Fluorescence and differential interference contrast (DIC) images were acquired and processed with FluoView FV1000 version 1.7.1.0 software.

\section{Results}

\subsection{Purification of DmCatD from eggs of D. maxima}

The activity of cathepsin D peptidase in egg homogenates from D. maxima was confirmed, in line with our previous report on the fat body, ovarian tissue and hemolymph (Leyria et al., 2015). Based on this result, purification of DmCatD was conducted by a combination of cation-exchange and gel filtration chromatography, employing homogenates of eggs collected within $24 \mathrm{~h}$ post oviposition as starting biological material. After the first chromatographic step, the activity of cathepsin D peptidase was found to be highest in the fraction eluted with $200 \mathrm{mM} \mathrm{NaCl}$ (data not shown). After gel filtration, a major peak of cathepsin D peptidase activity was eluted at $10.5 \mathrm{ml}$ (Fig. 1A). When this fraction was subjected to SDS-PAGE, a single band of approximately $43 \mathrm{kDa}$ was detected (Fig. 1B). As shown in Fig. 1C, the yield of purified DmCatD peptidase was $6.3 \%$ achieving 64 -fold of purification.

The $43 \mathrm{kDa}$ band obtained from gel filtration was excised from the gel, digested with trypsin and the resulting peptides were analyzed by MS/MS. When searched against the NCBI database, the peptide profiles matched the cathepsins D from D. maxima and T. infestans (Table 1). Three independent samples from different purification batches yield the same set of results.

\subsection{Sequence analysis and structural properties of DmCatD}

When the RT-PCR assays were performed to obtain the sequence of DmCatD, a single PCR product of the expected size and sequence was amplified. As expected, approximately $73 \%$ of full-length DmCatD transcript was obtained, sequenced and annotated in the GenBank under the ID KF724683.1. The protein has a predicted sequence of 284 amino acids (GenBank: AHE57676.1) (Fig. 2A). Since the N-terminus is necessary to perform specific bioinformatic analyses and the DmCatD sequence was not complete, the homologue region from $T$. infestans Cathepsin D was used to form a chimeric full-length sequence (amino acids in bold, Fig. 2B).

Bioinformatic analysis performed using NCBI tools showed that in the chimeric cathepsin D construction, the sequence corresponding to DmCatD presented a catalytic aspartic 
peptidase active site motif, DTGS, in the C-terminus lobe (Fig. 2B, underlined region) and displayed a conserved catalytic site of aspartate (Fig. 2B, arrow). In addition, DmCatD showed an extended loop, projecting over the cleft to form a flap of 11 residues in the active site (RLVYGKGSMVG, Fig. 2B, asterisks), which is conserved in cathepsin D family (Metcalf and Fusek, 1993). It was also found that in DmCatD, 5 of the 6 cysteine residues, which are characteristic of the family of aspartic peptidases (Shewale and Tang, 1984; Fusek and Větvičkab, 2005), were conserved (Fig. 2B, stars).

DmCatD also showed a post-translational cleavage site that varies in length and amino acid composition in different species (Fig. 2B, shaded regions) (Zaidi et al., 2008).

Bioinformatics approaches that included the prediction of putative phosphorylation and $\mathrm{N}$ myristoylation sites occurring in the DmCatD sequence are summarized in Fig. 2C. On the other hand, the structural properties of DmCatD peptidase were assessed in silico employing the chimeric construct that included the $\mathrm{N}$-terminus sequence of $T$. infestans cathepsin $\mathrm{D}$. The analysis of the structural model for DmCatD (Fig. 3A) indicated that the protein has a positively charged, hydrophilic surface as one of its main features (Fig. 3B-C, respectively).

\subsection{Molecular phylogenetic analysis of DmCatD}

According to the phylogenetic tree obtained upon the evolutionary analysis carried out with the DmCatD sequence deposited in NCBI database (ID KF724683.1), cathepsin D from the hemipterans D. maxima, T. infestans and Riptortus pedestris but not from Halyomorpha halys clustered together even though they are distantly related to the other sequences, including those from insects (Fig. 4). Cathepsin D from H. halys is shown as an intermediate between such a cluster and the other inspected sequences. Thus, cathepsin D from these four hemipteran species constitutes a unique group of enzymes, with peculiar features that suggest distancing from "classical" cathepsin D peptidases. Most of the insect sequences also clustered following their orders as can be seen for the hymenopterans Bombus impatiens, Melipona quadrifasciata, Apis florea, Apis dorsata, Apis mellifera, Polyrhachis vicina, Vollenhovia emeryi and Wasmannia auropunctata. However, the louse Pediculus humanus was placed together with the non-insect copepods Calugus clemensi and Lepeophtheirus salmonis. The sequences of the remaining non-insect groups analyzed, including bivalves, ticks and mammals also clustered together.

Alignment of the proline-loop region of all the sequences employed in the phylogenetic inference and the consensus sequence for this motif showed that cathepsin D from hemipterans had a nonconserved proline loop (Fig. 5).

\subsection{DmCatD and lipophorin interaction: co-immunoprecipitation, colocalization and docking analysis}

Taking into account the relevant function of DmCatD in the reproductive biology of $D$. maxima (Aguirre et al., 2011; Leyria et al., 2015) we performed approaches to address the role of lipophorin in carrying DmCatD to developing oocytes. Co-immunoprecipitation assays were carried out to test the interaction between DmCatD-lipophorin and DmCatDvitellogenin in the hemolymph of vitellogenic females of D. maxima. In our experimental conditions, the results showed that DmCatD co-immunoprecipitated with endogenous 
lipophorin but not with vitellogenin (Fig. 6A-B). Furthermore, the colocalization of DmCatD and lipophorin was investigated by immunofluorescence, analyzing vitellogenic follicles. Colocalization of lipophorin with DmCatD was detected in the perioocytic space, likely bound to the oocyte membrane, and in the yolk bodies (Fig. 7, merged images, upper panel). Negative controls corresponding to immunofluorescence assays show the specificity of the antibodies (Fig. 7, lower panel). Taken together, the results support the interaction between DmCatD and lipophorin in the hemolymph from vitellogenic females, and strongly suggest that such a lipoprotein acts as a DmCatD carrier cooperating with its targeting and posterior internalization by the oocytes.

In addition, computational assays were undertaken to analyze the feasibility of a binding between DmCatD and lipophorin. For that purpose, we modeled the lipophorin structure employing the apolipophorin II/I sequence from $R$. prolixus. The resulting model was then used to perform docking assays involving lipophorin and DmCatD. As shown in Fig. 8, the obtained complexes were convergent regarding their binding location and orientation. Since these are docking arrangements obtained from two independent software programs, without any spatial restriction for the docking search space, their common focal localization for the DmCatD-lipophorin complex formation highlights its higher probability as a physiological complex in the insect.

\section{Discussion}

Cathepsin D is a soluble lysosomal aspartic endopeptidase involved in the degradation and/or activation of proteins, hormones and enzymes, among other functions (Benes et al., 2008). Regarding triatomines, cathepsin $\mathrm{D}$ is implicated in yolk protein degradation during the embryonic development of $R$. prolixus (Fialho et al., 2005; Gomes et al., 2010) as well as in the digestion of ingested blood proteins in $T$. infestans (Balczun et al., 2012). In $D$. maxima, DmCatD peptidase was necessary for promoting early degradation of vitellin if follicular atresia was triggered by deprivation of blood meals (Aguirre et al., 2011; Leyria et al., 2015). In this work, we have obtained original information about DmCatD by achieving its purification from egg homogenates. By ion-exchange chromatography and gel filtration (Fig. 1A), a standard protocol for purifying cathepsin $\mathrm{D}$ as well as other peptidases (Defferrari et al., 2011), we have found that DmCatD from eggs displayed a molecular mass of $43 \mathrm{kDa}$ (Fig. 1B), similar to those reported for pro-DmCatD in the fat body and ovaries by western blots (Leyria et al., 2015). The MS/MS analysis of the $43 \mathrm{kDa}$ band obtained after purification retrieved peptide profiles matching those of the cathepsin D from D. maxima (AHE57676) and T. infestans (AEO94539), confirming thus its identity (Table 1).

In this work, the predicted protein sequence of DmCatD (AHE57676.1) shares an $86 \%$ identity with cathepsin D protein of $T$. infestans (ADK47877.1) (Fig. 2). On the other hand, a pro-cathepsin of $\sim 42 \mathrm{kDa}$ molecular mass in the chimeric cathepsin $\mathrm{D}$ construction is close to the mass of the purified DmCatD from eggs and MS/MS analysis reported here (Fig. 1), and it is also in agreement with the pro-DmCatD described in Leyria et al. (2015) and to the findings in other insects (Padilha et al. 2009; Gui et al. 2006; Kang et al. 2017). 
Bioinformatic analysis revealed that the chimeric cathepsin D displays a conserved catalytic site consisting in two aspartate residues, one of them found in the DmCatD sequence (Fig. 2B). These aspartate residues are known to play a key catalytic role in cathepsins D and E of the pepsin family as well as in renins (Dunn, 2002; Fusek and Větvička, 2005). On the contrary, the aspartic peptidase BYC from the tick Rhipicephalus (Boophilus) microplus eggs, which is responsible for the yolk degradation during embryogenesis, lacks the second aspartate residue from the catalytic site (Nascimiento-Silva et al., 2008). Eukaryotic aspartic peptidases contain two conserved DTGS/T motifs, each one provides a catalytic aspartate residue to the active site (Davies, 1990). In DmCatD peptidase sequence, the DTGS motif in the C-terminus lobe was strictly conserved (Fig. 2B)

The deduced DmCatD peptidase sequence showed putative phosphorylation and Nmyristoylation sites (Fig. 2C), that could be relevant in the post-translational modification of the protein and, consequently, in the regulation of its binding and enzymatic properties.

The structural model for the chimeric construct of cathepsin D assessed in silico indicated that this peptidase exhibited a positively charged, hydrophilic surface as main features (Fig. 3 ), being the latter a characteristic expected for a soluble protein. These properties might contribute to its interaction with lipophorin, discussed below, which in turn has predominantly a negative surface charge (Roosendaal et al., 2009). However, the contribution of the hydrophobic and negative patches of DmCatD surface to the binding capacity of the lipoprotein cannot be disregarded (Smith and Davidson, 2010).

Molecular phylogenetic analysis from selected taxa indicated that in Hemiptera, cathepsin D clusters together but not in the expected pattern from species-level phylogeny (Fig. 4). Within the cladogram, hemipteran cathepsin D is clearly separated from that of other insects and vertebrates. This clustering pattern has been observed recently in a survey for cathepsin D from the midgut of the hemipteran Dysdercus peruvianus (Pimentel et al., 2017). The main reason for this separation seems to be the non-lysosomal nature of the majority of cathepsin D peptidases in hemipterans, as indicated by their non-conserved proline loop (Fig. 5). In Musca domestica, three cathepsin D peptidases were reported in the midgut, two of them lacking the proline loop. It was suggested that this characteristic could be somehow associated with an extracellular role for these enzymes (Padilha et al., 2009). In the hemipteran $D$. peruvianus, nine of ten cathepsin $\mathrm{D}$ transcribing genes that were specifically expressed in the midgut lack the proline loop (Pimentel et al., 2017). Additionally, two cathepsins D having or not a proline loop, were found to be secreted into the midgut lumen of T. infestans (Balczun et al., 2012). The authors proposed that such a finding could be an adaptation limited to triatomines. In this work, the results indicated that DmCatD displayed features in common with secreted peptidases. Moreover, we recently reported that DmCatD was localized in yolk bodies, which are specialized compartments in developing oocytes (Leyria et al., 2015). These findings can be related to the physiological role of DmCatD assigned in D. maxima, which functions as a yolk protein precursor during vitellogenesis but also as an acid peptidase that regulates vitellin degradation.

We have reported that $\mathrm{DmCatD}$, which is synthesized as a yolk protein precursor in the fat body and the ovarian tissue, is involved in vitellin degradation during follicular atresia 
(Leyria et al., 2015). Studies in A. aegypti demonstrated that a vitellogenic carboxypeptidase (VCB) and a thiol cathepsin B pro-protease (VCP), which are both involved in yolk degradation during embryogenesis, are synthesized in the fat body as yolk protein precursors and stored in developing oocytes along with vitellin (Cho et al., 1991; 1999). However, how these yolk protein precursors are targeted from the fat body to oocytes through the hemolymph and further internalized remains to be elucidated. Swevers et al. (2005) proposed that lipophorin serves as a carrier for VCB and VCP, and that the internalization of these yolk protein precursors probably occurs via a "piggyback" mechanism. Up to date, no experimental evidence supporting this pathway was reported.

Lipophorin mostly delivers its hydrophobic cargo to developing oocytes by its binding to non-endocytic receptors at the plasma membrane of the cells (Ziegler and Van Antwerpen, 2006). However, in certain insects including at least two triatomine species, lipophorin also functions as a yolk protein, being endocytosed by the oocytes, stored in yolk bodies and used to support embryonic development (Kawooya and Law, 1988; Ziegler and Van Antwerpen, 2006; Fruttero et al., 2011, 2017; Leyria et al., 2014). In this context, it has been reported that lipophorin receptor ( $\mathrm{LpR}$ ), which belongs to the low-density lipoprotein receptor (LDLR) gene superfamily, when it is expressed on the oocyte membranes as well as in other tissues mediates the internalization of lipophorin particles. Taking into account the relevant role of DmCatD in the reproductive biology of D. maxima (Aguirre et al., 2011; Leyria et al., 2015) and considering that the mechanism involved in its targeting to developing oocytes is unknown, we performed experiments to address the role of lipophorin as a carrier of DmCatD in vitellogenic females. Co-immunoprecipitation assays (Fig. 6) and colocalization of lipophorin and DmCatD (Fig. 7) support their endogenous association. The association between cathepsin $\mathrm{D}$ and a lipoprotein has been described previously. Thus, it was reported that cathepsin D associated with both, lipid-free recombinant full-length human apolipoprotein $\mathrm{E}$ and lipidated human plasma full-length apolipoprotein $\mathrm{E}$, playing a possible role in Alzheimer's disease (Zhou et al., 2006). Additionally, the results from computational docking assays favored the lipophorin-DmCatD interaction (Fig. 8).

From a physiological point of view, this work provides evidence indicating that lipophorin acts as a carrier for DmCatD, which in turn could be part of a mechanism of functional relevance for its transport to developing oocytes and further internalization. In contrast to lipophorin, which binds to several molecules such as proteins and hormones (Ma et al., 2006; Rahman et al., 2006; Zalewska et al., 2009), vitellogenin has been reported to interact with few partners (Engelmann and Mala, 2000). Therefore, it seems very unlikely that vitellogenin participates in the transport of DmCatD through circulation since under our experimental conditions no co-immunoprecipitation between these proteins in the hemolymph of vitellogenic females was observed. On the other hand, even though hemolymph $\mathrm{pH}$ is always near neutrality (Harrison, 2001), the microenvironment associated to the extracellular side of the plasma membrane may exhibit $\mathrm{pH}$ changes (Maouyo et al., 2000 ) that could lead to cathepsin D activation. Thus, binding of lipophorin with DmCatD may also be important in preserving such a peptidase from both activation and/or proteolytic events in the hemolymph. 
Until now, the mechanisms involved in the internalization of peptidases within the oocytes of insects have not been reported. In mammals, intracellular transport of pro-cathepsin D is mediated by the mannose-6-phosphate receptors (M6Pr), which capture and target the proenzyme to lysosomal compartments (Fusek and Větvička, 2005). However, alternative M6Pr-independent mechanisms have also been reported (Laurent-Matha et al., 1998). For instance, in mammary human fibroblasts, secreted pro-cathepsin D is partly endocytosed by LDL receptor-related protein-1 (LRP1) (Derocq et al., 2012). This finding suggests a role of $\mathrm{LpR}$ in the endocytosis of pro-DmCatD since both, LRP and LpR are members of the LDL receptor family (Ziegler and Van Antwerpen, 2006). Furthermore, the colocalization of lipophorin and DmCatD in yolk bodies (Fig. 7) also suggests the LpR participation. It is also important to analyze if the non-endocytic lipophorin receptor, the $\beta$ subunit of the ATP synthase complex ( $\beta$-ATPase), which is expressed in the oocytes of a related triatomine Panstrongylus megistus (Fruttero et al., 2017), contributes in docking the lipophorinDmCatD complex at the oocyte membrane as a step for its further internalization.

In summary, DmCatD is part of a physiological mechanism that regulates yolk protein degradation in D. maxima (Leyria et al., 2015). The interaction of DmCatD with lipophorin demonstrated in this work offers a unique scenario to unveil the participation of different lipophorin receptors, which in turn may modulate each other or function in coordination by promoting the internalization of DmCatD by developing oocytes.

\section{Acknowledgments}

We thank Biol. Raúl Stariolo (Coordinación Nacional de Control de Vectores, Córdoba, Argentina) as well as Mr. F. Navarro and Mr. D. Luti (CIBICI-CONICET-UNC) for assistance in animal care. Authors also thank Melissa Postal, Fernanda Cortez Lopes and Diogo Ribeiro Demartini (Department of Molecular Biology and Biotechnology, UFRGS, Porto Alegre, Brazil) for laboratory technical support. This work was supported by Grants from FONCyT (PICT 2013-0626, ANPCyT-Argentina), Consejo Nacional de Investigaciones Científicas y Técnicas (CONICET, PIP 0159) and SECyT-Universidad Nacional de Córdoba (PID C05/456). LEC and BPS are established researchers of CIC-CONICET, Argentina. JL is a postdoctoral research fellow from CONICET. The authors would like to thank both reviewers for their insightful comments that greatly contributed to improving the final version of the paper.

\section{Abbreviations}

$\begin{array}{ll}\beta \text {-ATPase } & \beta \text {-chain of ATP synthase } \\ \text { OCT } & \text { Optimal Cutting Temperature } \\ \text { BSA } & \text { Bovine serum albumin } \\ \text { DMP } & \text { dimethylpimelimidate } \\ \text { FBS } & \text { fetal bovine serum } \\ \text { PMSF } & \text { phenylmethyl-sulfonyl fluoride } \\ \text { TLCK } & N \text {-a-p-tosyl-L-lysine chloromethyl ketone } \\ \text { PBS } & \text { phosphate buffered saline } \\ \text { NaPB } & \text { sodium phosphate buffer }\end{array}$


SDS-PAGE sodium dodecyl sulfate-polyacrylamide gel electrophoresis

MS/MS tandem mass spectrometry

RT-PCR Reverse Transcription - Polymerase chain reaction

TBS Tris buffered saline

anti-Lp anti-lipophorin antibody

anti-Vt anti-vitellin antibody

FITC fluorescein isothiocyanate

LPR lipophorin receptor

LDLR low-density lipoprotein receptor

M6Pr mannose-6-phosphate receptor

LRP1 LDL receptor-related protein-1

Co-IP co-immunoprecipitation

Lp lipophorin

Vg vitellogenin

\section{References}

Aguirre SA, Frede S, Rubiolo ER, Canavoso LE. Vitellogenesis in the hematophagous Dipetalogaster maxima (Hemiptera: Reduviidae), a vector of Chagas' disease. J Insect Physiol. 2008; 54:393-402. [PubMed: 18068184]

Aguirre SA, Fruttero LL, Leyria J, Defferrari MS, Pinto PM, Settembrini BP, Rubiolo ER, Carlini CR, Canavoso LE. Biochemical changes in the transition from vitellogenesis to follicular atresia in the hematophagous Dipetalogaster maxima (Hemiptera: Reduviidae). Insect Biochem Mol Biol. 2011; 42:832-841.

Artimo P, Jonnalagedda M, Arnold K, Baratin D, Csardi G, de Castro E, Duvaud S, Flegel V, Fortier A, Gasteiger E, Grosdidier A, Hernandez C, Ioannidis V, Kuznetsov D, Liechti R, Moretti S, Mostaguir K, Redaschi N, Rossier G, Xenarios I, Stockinger H. ExPASy: SIB bioinformatics resource portal. Nucleic Acids Res. 2012; 40:W597-603. [PubMed: 22661580]

Atella GC, Gondim KC, Machado EA, Medeiros MN, Silva-Neto MA, Masuda H. Oogenesis and egg development in triatomines: a biochemical approach. An Acad Bras Cienc. 2005; 77:405-430. [PubMed: 16127549]

Baker NA, Sept D, Joseph S, Holst MJ, McCammon JA. Electrostatics of nanosystems: application to microtubules and the ribosome. Proc Natl Acad Sci USA. 2001; 98:10037-10041. [PubMed: 11517324]

Balczun C, Siemanowski J, Pausch JK, Helling S, Marcus K, Stephan C, Meyer HE, Schneider T, Cizmowski C, Oldenburg M, Höhn S, Meiser CK, Schuhmann W, Schaub GA. Intestinal aspartate proteases TiCatD and TiCatD2 of the haematophagous bug Triatoma infestans (Reduviidae): sequence characterisation, expression pattern and characterisation of proteolytic activity. Insect Biochem Mol Biol. 2012; 42:240-250. [PubMed: 22210150]

Benes P, Vetvicka V, Fusek M. Cathepsin D: many functions of one aspartic protease. Crit Rev Oncol Hematol. 2008; 68:12-28. [PubMed: 18396408] 
Blom N, Gammeltoft S, Brunak S. Sequence and structure-based prediction of eukaryotic protein phosphorylation sites. J Mol Biol. 1999; 294:1351-1362. [PubMed: 10600390]

Bradford MM. A rapid and sensitive method for the quantitation of micrograms quantities of protein utilizing the principle of protein-dye binding. Anal Biochem. 1976; 72:248-254. [PubMed: 942051]

Canavoso LE, Rubiolo ER. Interconversions of lipophorin particles by adipokinetic hormone in hemolymph of Panstrongylus megistus, Dipetalogaster maximus and Triatoma infestans (Hemiptera: Reduviidae). Comp Biochem Physiol A. 1995; 112:143-150.

Canavoso LE, Jouni ZE, Karnas KJ, Pennington JE, Wells MA. Fat metabolism in insects. Annu Rev Nutr. 2001; 21:23-46. [PubMed: 11375428]

Cho WL, Deitsch KW, Raikhel AS. An extraovarian protein accumulated in mosquito oocytes is a carboxypeptidase activated in embryos. Proc Natl Acad Sci USA. 1991; 88:10821-10824. [PubMed: 1961751]

Cho WL, Tsao SM, Hays AR, Walter R, Chen JS, Snigirevskaya ES, Raikhel AS. Mosquito cathepsin B-like protease involved in embryonic degradation of vitellin is produced as a latent extraovarian precursor. J Biol Chem. 1999; 274:13311-13321. [PubMed: 10224092]

Comeau SR, Gatchell DW, Vajda S, Camacho CJ. ClusPro: A fully automated algorithm for proteinprotein docking. Nucleic Acids Res. 2004; 32:W96-W99. [PubMed: 15215358]

Davies DR. The structure and function of the aspartic proteinases. Annu Rev Biophys Biophys Chem. 1990; 19:189-215. [PubMed: 2194475]

Defferrari MS, Demartini DR, Marcelino TB, Pinto PM, Carlini CR. Insecticidal effect of Canavalia ensiformis major urease on nymphs of the milkweed bug Oncopeltus fasciatus and characterization of digestive peptidases. Insect Biochem Mol Biol. 2011; 41:388-399. [PubMed: 21377528]

Derocq D, Prébois C, Beaujouin M, Laurent-Matha V, Pattingre S, Smith GK, Liaudet-Coopman E. Cathepsin D is partly endocytosed by the LRP1 receptor and inhibits LRP1-regulated intramembrane proteolysis. Oncogene. 2012; 31:3202-3212. [PubMed: 22081071]

Dunn BM. Structure and mechanism of the pepsin-like family of aspartic peptidases. Chem Rev. 2002; 102:4431-4458. [PubMed: 12475196]

Engelmann F, Mala J. The interactions between juvenile hormone ( $\mathrm{JH})$, lipophorin, vitellogenin, and JH esterases in two cockroach species. Insect Biochem Mol Biol. 2000; 30:793-803. [PubMed: 10876123]

Eugster C, Panáková D, Mahmoud A, Eaton S. Lipoprotein-heparan sulfate interactions in the Hh pathway. Dev Cell. 2007; 13:57-71. [PubMed: 17609110]

Fialho E, Nakamura A, Juliano L, Masuda H, Silva-Neto MA. Cathepsin D-mediated yolk protein degradation is blocked by acid phosphatase inhibitors. Arch Biochem Biophys. 2005; 436:246253. [PubMed: 15797237]

Fruttero LL, Rubiolo ER, Canavoso LE. Biochemical and cellular characterization of lipophorinmidgut interaction in the hematophagous Panstrongylus megistus (Hemiptera: Reduviidae). Insect Biochem Mol Biol. 2009; 9:322-331.

Fruttero LL, Frede S, Rubiolo ER, Canavoso LE. The storage of nutritional resources during vitellogenesis of Panstrongylus megistus (Hemiptera: Reduviidae): the pathways of lipophorin in lipid delivery to developing oocytes. J Insect Physiol. 2011; 57:475-486. [PubMed: 21277855]

Fruttero LL, Demartini DR, Rubiolo ER, Carlini CR, Canavoso LE. $\beta$-chain of ATP synthase as a lipophorin binding protein and its role in lipid transfer in the midgut of Panstrongylus megistus (Hemiptera: Reduviidae). Insect Biochem Mol Biol. 2014; 52:1-12. [PubMed: 24952172]

Fruttero LL, Leyria J, Ramos FO, Stariolo R, Settembrini BP, Canavoso LE. The process of lipid storage in insect oocytes: The involvement of $\beta$-chain of ATP synthase in lipophorin-mediated lipid transfer in the Chagas' disease vector Panstrongylus megistus (Hemiptera: Reduviidae). J Insect Physiol. 2017; 96:82-92. [PubMed: 27983943]

Fusek M, Větvička V. Dual role of cathepsin D: ligand and protease. Biomed Papers. 2005; 149:43-50.

Giorgi, F., Nordin, JH. Biochemical and ultrastructural aspects of vitellin utilization during embryogenesis. In: Raikhel, AS., editor. Progress in vitellogenesis. Reproductive biology of invertebrates. Vol. 12. USA: Science Publishers; 2005. p. 355-391. 
Gomes FM, Oliveira DM, Motta LS, Ramos IB, Miranda KM, Machado EA. Inorganic polyphosphate inhibits an aspartic protease-like activity in the eggs of Rhodnius prolixus (Stahl) and impairs yolk mobilization in vitro. J Cell Physiol. 2010; 222:606-611. [PubMed: 19957302]

Gui ZZ, Lee KS, Kim BY, Choi YS, Wei YD, Choo YM, Kang PD, Yoon HJ, Kim I, Je YH, Seo SJ, Lee SM, Guo X, Sohn HD, Jin BR. Functional role of aspartic proteinase cathepsin D in insect metamorphosis. BMC Dev Biol. 2006; 6:49. [PubMed: 17062167]

Harrison JF. Insect acid-base physiology. Annu Rev Entomol. 2001; 46:221-250. [PubMed: 11112169]

Hotez PJ, Bottazzi ME, Franco-Paredes C, Ault SK, Periago MR. The neglected tropical diseases of Latin America and the Caribbean: A review of disease burden and distribution and a roadmap for control and elimination. PLoS Negl Trop Dis. 2008; 2:e300. [PubMed: 18820747]

Izumi S, Yano K, Yamamoto Y, Takahashi SY. Yolk proteins from insect eggs: structure, biosynthesis and programmed degradation during embryogenesis. J Insect Physiol. 1994; 40:735-746.

Johnson M, Zaretskaya I, Raytselis Y, Merezhuk Y, McGinnis S, Madden TL. NCBI BLAST: a better web interface. Nucleic Acids Res. 2008; 36:W5-W9. [PubMed: 18440982]

Kang T, Jin R, Zhang Y, Wan H, Lee KS, Jin BR, Li J. Functional characterization of the aspartic proteinase cathepsin D in the beet armyworm (Spodoptera exigua). Gene. 2017; 617:1-7. [PubMed: 28351737]

Kawooya JK, Law JH. Role of lipophorin in lipid transport to the insect egg. J Biol Chem. 1988; 263:8748-8753. [PubMed: 3379043]

Kelley LA, Mezulis S, Yates CM, Wass MN, Sternberg MJ. The Phyre2 web portal for protein modeling, prediction and analysis. Nat Protoc. 2015; 10:845-858. [PubMed: 25950237]

Kozakov D, Brenke R, Comeau SR, Vajda S. PIPER: An FFT-based protein docking program with pairwise potentials. Proteins. 2006; 65:392-406. [PubMed: 16933295]

Kyte J, Doolittle RF. A simple method for displaying the hydropathic character of a protein. J Mol Biol. 1982; 157:105-132. [PubMed: 7108955]

Laemmli UK. Cleavage of structural proteins during the assembly of the head of bacteriophage T4. Nature. 1970; 227:680-685. [PubMed: 5432063]

Laskowski RA, MacArthur MW, Moss DS, Thornton JM. PROCHECK: a program to check the stereochemical quality of protein structures. J Appl Cryst. 1993; 26:283-291.

Laurent-Matha V, Farnoud MR, Lucas A, Rougeot C, Garcia M, Rochefort H. Endocytosis of procathepsin D into breast cancer cells is mostly independent of mannose-6-phosphate receptors. J Cell Sci. 1998; 111:2539-2549. [PubMed: 9701553]

Le SQ, Gascuel O. An improved general amino acid replacement matrix. Mol Biol Evol. 2008; 25:1307-1320. [PubMed: 18367465]

Leyria J, Fruttero LL, Aguirre SA, Canavoso LE. Ovarian nutritional resources during the reproductive cycle of the hematophagous Dipetalogaster maxima (Hemiptera: Reduviidae): focus on lipid metabolism. Arch Insect Biochem Physiol. 2014; 87:148-163. [PubMed: 25052220]

Leyria J, Fruttero LL, Nazar M, Canavoso LE. The role of DmCatD, a cathepsin D-like peptidase, and acid phosphatase in the process of follicular atresia in Dipetalogaster maxima (Hemiptera: Reduviidae), a vector of Chagas' disease. PLoS One. 2015; 10:e0130144. [PubMed: 26091289]

Ma G, Hay D, Li D, Asgari S, Schmidt O. Recognition and inactivation of LPS by lipophorin particles. Dev Comp Immunol. 2006; 30:619-626. [PubMed: 16386790]

Maouyo D, Chu S, Montrose MH. pH heterogeneity at intracellular and extracellular plasma membrane sites in HT29-C1 cell monolayers. Am J Physiol Cell Physiol. 2000; 278:C973-C981. [PubMed: 10794671]

Melo AC, Valle D, Machado EA, Salerno AP, Paiva-Silva GO, Cunha E, Silva NL, de Souza W, Masuda H. Synthesis of vitellogenin by the follicle cells of Rhodnius prolixus. Insect Biochem Mol Biol. 2000; 30:549-557. [PubMed: 10844247]

Metcalf P, Fusek M. Two crystal structures for cathepsin D: the lysosomal targeting signal and active site. EMBO J. 1993; 12:1293-1302. [PubMed: 8467789]

Miles MA. Chagas Disease (American Trypanosomiasis). Infectious diseases (Fourth Edition). 2017; 2:1065-1072. 
Nascimento-Silva MC, Leal AT, Daffre S, Juliano L, da Silva Vaz I Jr, Paiva-Silva GO, Oliveira PL, Sorgine MH. BYC, an atypical aspartic endopeptidase from Rhipicephalus (Boophilus) microplus eggs. Comp Biochem Physiol B Biochem Mol Biol. 2008; 149:599-607. [PubMed: 18242110]

Núñez, JA., Segura, EL. Rearing of triatominae. In: Brenner, RR., Stoka, AM., editors. Chagas' disease vectors. Vol. 2. Florida: CRC Press; 1987. p. 31-40.

Nussenzveig RH, Oliveira PL, Masuda H. Identification of yolk platelet-associated hydrolases in the oocytes of Rhodnius prolixus. Arch Insect Biochem Physiol. 1992; 21:253-262. [PubMed: 1286200]

Oliveira DM, Ramos IB, Reis FC, Lima AP, Machado EA. Interplay between acid phosphatase and cysteine proteases in mediating vitellin degradation during early embryogenesis of Periplaneta americana. J Insect Physiol. 2008; 54:883-891. [PubMed: 18499122]

Padilha MHP, Pimentel AC, Ribeiro AF, Terra WR. Sequence and function of lysosomal and digestive cathepsin D-like proteinases of Musca domestica midgut. Insect Biochem Mol Biol. 2009; 39:782791. [PubMed: 19815068]

Petersen TN, Brunak S, von Heijne G, Nielsen H. SignalP 4.0: discriminating signal peptides from transmembrane regions. Nat Methods. 2011; 8:785-786. [PubMed: 21959131]

Pettersen EF, Goddard TD, Huang CC, Couch GS, Greenblatt DM, Meng EC, Ferrin TE. UCSF Chimera - a visualization system for exploratory research and analysis. J Comput Chem. 2004; 25:1605. [PubMed: 15264254]

Pimentel AC, Fuzita FJ, Palmisano G, Ferreira C, Terra WR. Role of cathepsins D in the midgut of Dysdercus peruvianus. Comp Biochem Physiol B Biochem Mol Biol. 2017; 204:45-52. [PubMed: 27838410]

Rahman MM, Ma G, Roberts HL, Schmidt O. Cell-free immune reactions in insects. J Insect Physiol. 2006; 52:754-762. [PubMed: 16753175]

Raikhel AS, Dhadialla TS. Accumulation of yolk proteins in insect oocytes. Annu Rev Entomol. 1992; 37:217-251. [PubMed: 1311540]

Raikhel, AS. Vitellogenesis of disease vectors, from physiology to genes. In: Marquardt, WC., editor. Biology of disease vectors. Elsevier Academic Press; London, UK: 2005. p. 329-346.

Roosendaal SD, Van Doorn JM, Valentijn KM, Van der Horst DJ, Rodenburg KW. Delipidation of insect lipoprotein, lipophorin, affects its binding to the lipophorin receptor, LpR: implications for the role of LpR-mediated endocytosis. Insect Biochem Mol Biol. 2009; 39:135-144. [PubMed: 19049873]

Roy A, Kucukural A, Zhang Y. I-TASSER: a unified platform for automated protein structure and function prediction. Nat Protoc. 2010; 5:725-738. [PubMed: 20360767]

Schneidman-Duhovny D, Inbar Y, Nussinov R, Wolfson HJ. PatchDock and SymmDock: servers for rigid and symmetric docking. Nucleic Acids Res. 2005; 33:W363-W367. [PubMed: 15980490]

Shewale JG, Tang J. Amino acid sequence of porcine spleen cathepsin D. Proc Natl Acad Sci USA. 1984; 81:3703-3707. [PubMed: 6587385]

Sigrist CJA, de Castro E, Cerutti L, Cuche BA, Hulo N, Bridge A, Bougueleret L, Xenarios I. New and continuing developments at PROSITE. Nucleic Acids Res. 2013; 41:D344-D347. [PubMed: 23161676]

Smith LE, Davidson WS. The role of hydrophobic and negatively charged surface patches of lipid-free apolipoprotein A-I in lipid binding and ABCA1-mediated cholesterol efflux. Biochim Biophys Acta. 2010; 1801:64-69. [PubMed: 19782154]

Stoka, AM., Salomón, OD., Noriega, FG. Physiology of triatominae's reproduction. In: Brenner, RB., Stoka, AM., editors. Chagas' disease vectors. Vol. 2. CRC Press; Florida: 1987. p. 109-129.

Swevers, L., Raikhel, AS., Sappington, TW., Shirk, P., Iatrou, K. Vitellogenesis and post-vitellogenic maturation of the insect ovarian follicle. In: Gilbert, LI.Iatrou, K., Gill, SS., editors. Comprehensive Molecular Insect Science. Vol. 1. 2005. p. 87-155.

Tamura K, Stecher G, Peterson D, Filipski A, Kumar S. MEGA6: Molecular evolutionary genetics analysis version 6.0. Mol Biol Evol. 2013; 30:2725-2729. [PubMed: 24132122]

Tufail M, Takeda M. Molecular characteristics of insect vitellogenins. J Insect Physiol. 2008; 54:14471458. [PubMed: 18789336] 
Tufail M, Takeda M. Insect vitellogenin/lipophorin receptors: molecular structures, role in oogenesis, and regulatory mechanisms. J Insect Physiol. 2009; 55:87-103. [PubMed: 19071131]

Winnebeck EC, Millar CD, Warman GR. Why does insect RNA look degraded? J Insect Sci. 2010; 10:159. [PubMed: 21067419]

World Health Organization. [Accessed 2017 Jul 13] Chagas disease (American trypanosomiasis). Media centre -Fact sheets. Updated March 2017. Available: http://who.int/mediacentre/factsheets/ fs340/en/

Yamahama, Y., Yamamoto, Y., Watabe, S., Takahashi, SY. Regulation of yolk protein degradation during insect embryogenesis. In: Raikhel, AS., editor. Progress in vitellogenesis. Reproductive biology of invertebrates. Vol. 12. USA: Science Publishers; 2005. p. 315-354.

Yamamoto Y, Takahashi SY. Cysteine proteinase from Bombyx eggs: role in programmed degradation of yolk proteins during embryogenesis. Comp Biochem Physiol B. 1993; 106:35-45. [PubMed: 8403852]

Yang J, Yan R, Roy A, Xu D, Poisson J, Zhang Y. The I-TASSER Suite: protein structure and function prediction. Nat Methods. 2015; 12:7-8. [PubMed: 25549265]

Zaidi N, Maurer A, Nieke S, Kalbacher H. Cathepsin D: a cellular roadmap. Biochem Biophys Res Commun. 2008; 376:5-9. [PubMed: 18762174]

Zalewska M, Kochman A, Estève JP, Lopez F, Chaoui K, Susini C, Ozyhar A, Kochman M. Juvenile hormone binding protein traffic -- Interaction with ATP synthase and lipid transfer proteins. Biochim Biophys Acta. 2009; 1788:1695-705. [PubMed: 19426705]

Zhang Y. I-TASSER server for protein 3D structure prediction. BMC Bioinformatics. 2008; 9:40. [PubMed: 18215316]

Zhou W, Scott SA, Shelton SB, Crutcher KA. Cathepsin D-mediated proteolysis of apolipoprotein E: possible role in Alzheimer's disease. Neuroscience. 2006; 143:689-701. [PubMed: 16997486]

Ziegler R, Van Antwerpen R. Lipid uptake by insect oocytes. Insect Biochem Mol Biol. 2006; 36:264272. [PubMed: 16551540] 
Purification of DmCatD from $D$. maxima eggs

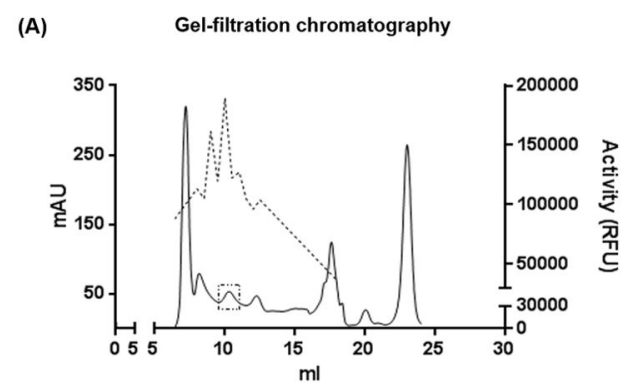

(B)

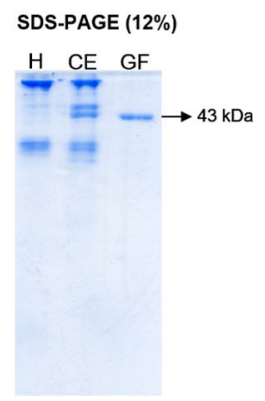

(C)

\begin{tabular}{|lcccccccc|}
\hline \multicolumn{1}{|c|}{ Steps of DmCatD purification } \\
\hline $\begin{array}{l}\text { Volume } \\
(\mathrm{ml})\end{array}$ & $\begin{array}{c}\text { Protein } \\
{[\mathrm{mg} / \mathrm{ml}]}\end{array}$ & $\begin{array}{c}\text { Total protein } \\
{[\mathrm{mg}]}\end{array}$ & $\begin{array}{c}\text { Activity } \\
{[\mathrm{RFU} / \mathrm{ml}]}\end{array}$ & $\begin{array}{c}\text { Specific } \\
\text { activity } \\
{[\mathrm{RFU} / \mathrm{mg}]}\end{array}$ & $\begin{array}{c}\text { Total activity } \\
{[\mathrm{RFU}]}\end{array}$ & $\begin{array}{c}\text { Vield } \\
{[\%]}\end{array}$ & Fold purified \\
\hline Homogenate & 6 & 10,52 & 63,12 & 807900 & 767968 & 48474000 & 100 & 1 \\
\hline CM-Sepharose & 20 & 0,079 & 1,58 & 1028257 & 13015913 & 20565143 & 42,4 & 1,7 \\
\hline Superdex 75 & 2 & 0,031 & 0,062 & 1523371 & 49141016 & 3046743 & 6,3 & 64 \\
\hline
\end{tabular}

Fig. 1.

Purification of cathepsin D (DmCatD) from homogenates of D. maxima eggs. (A), Gelfiltration chromatography profile on Superdex 75 equilibrated with $20 \mathrm{mM} \mathrm{NaPB}, \mathrm{pH} 6.0$, obtained after loading the active pool collected from the CM-Sepharose column. Mili absorbance units $\left(\mathrm{A}_{280}\right)$ are shown as a continuous line and the enzymatic activity of fractions upon Abz-AIAFFSRQ-EDDnp substrate as a dotted line. Protein peaks were assayed for enzymatic activity at $37^{\circ} \mathrm{C}$ for $30 \mathrm{~min}, 20 \mathrm{mM}$ citrate-phosphate buffer (pH 3.5). The major peak of enzymatic activity corresponds to the peak in the box. (B), SDSPAGE (12\%) corresponding to different steps of the purification protocol. H: Homogenate, CE: cation exchange active fraction (eluted at $200 \mathrm{mM} \mathrm{NaCl}$ ), GF: gel filtration fraction eluted at $10.5 \mathrm{ml}$. (C), Table of cathepsin D purification. Enzymatic activity of samples was assayed as stated in (A) and shown as Relative Fluorescence Units. CM-Sepharose (fraction eluted at $200 \mathrm{mM} \mathrm{NaCl}$ ); Superdex 75 (fraction eluted at $10.5 \mathrm{ml}$ ). 


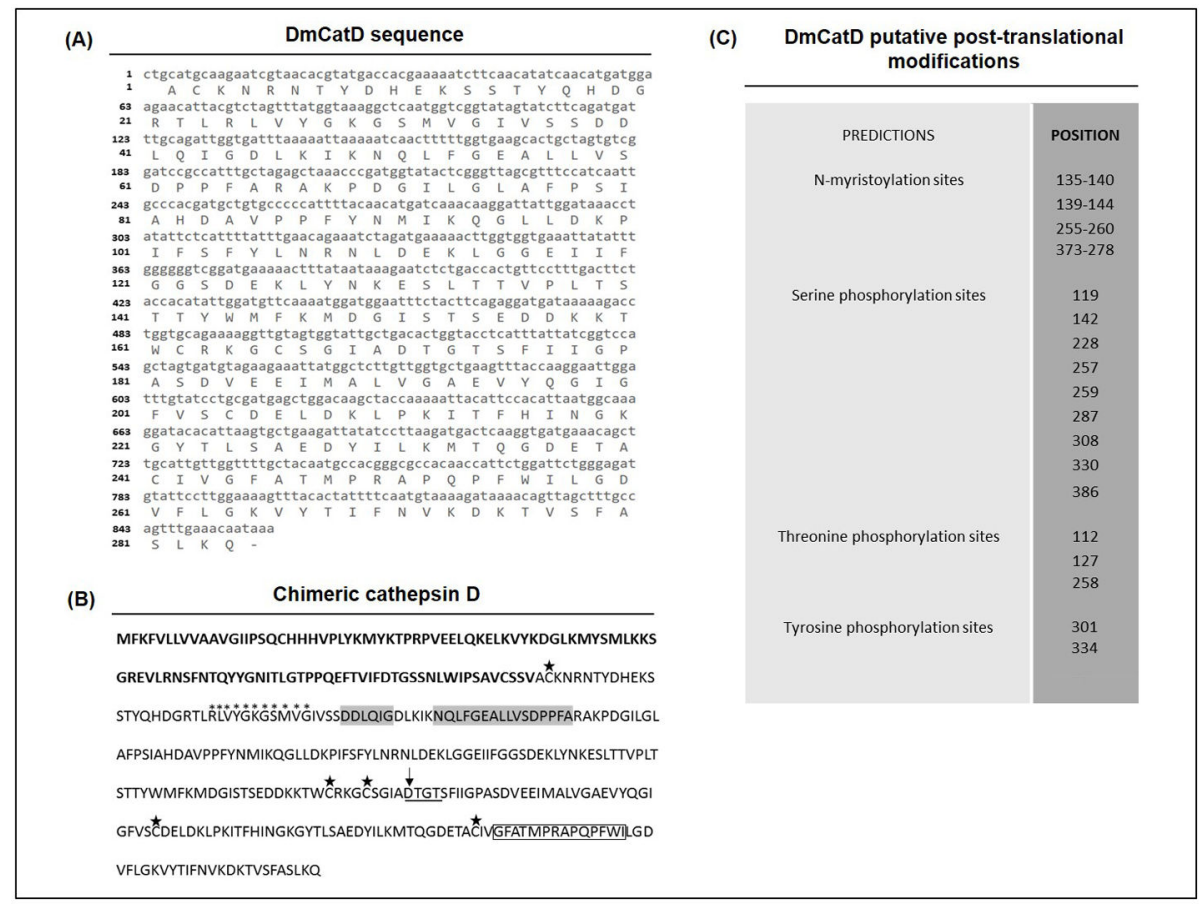

Fig. 2.

(A), Nucleotide and the corresponding deduced partial amino acid sequences of DmCatD. (B), In order to perform bioinformatic studies, the homologue region from Triatoma infestans cathepsin D was used to complete the $\mathrm{N}$-terminal region of the sequence (amino acids in bold). An arrow indicates the catalytic residue in the active site; underlined regions show a catalytic motif of the active site; asterisks indicate an active site flap; the posttranslational cleavage site is shown between the two highlighted blocks; the stars indicate the cysteine residues and the white block shows the proline-loop region. $(\mathbf{C})$, Putative phosphorylation and $\mathrm{N}$-myristoylation sites. 
(A)
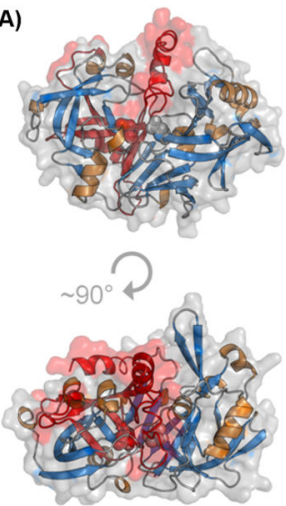

(B)
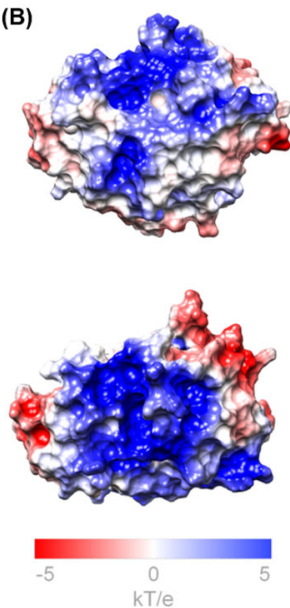

(C)
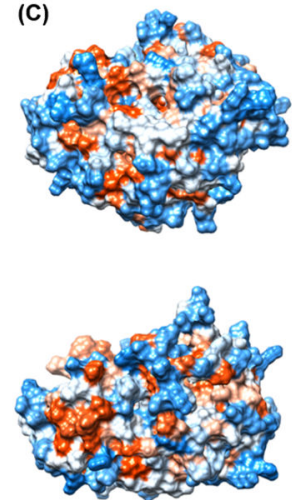

hydrophobic hydrophilic

Fig. 3.

(A), Structural model for the chimeric cathepsin D construct, shown as molecular surface superposed to a cartoon representation. The aspartic residues of the active site are shown as spheres, while the homologue region from T. infestans cathepsin D (used to build the chimeric full structure) is highlighted in red. (B), Electrostatic properties of cathepsin D, colored according to the electrostatic potential (red, negative; blue, positive). (C), Hydrophobicity properties of cathepsin D, colored according to the Kyte-Doolittle scale (orange, hydrophobic; light blue, hydrophilic). For all cases, the protein is depicted from the N-terminus (left) to C-terminus (right). 


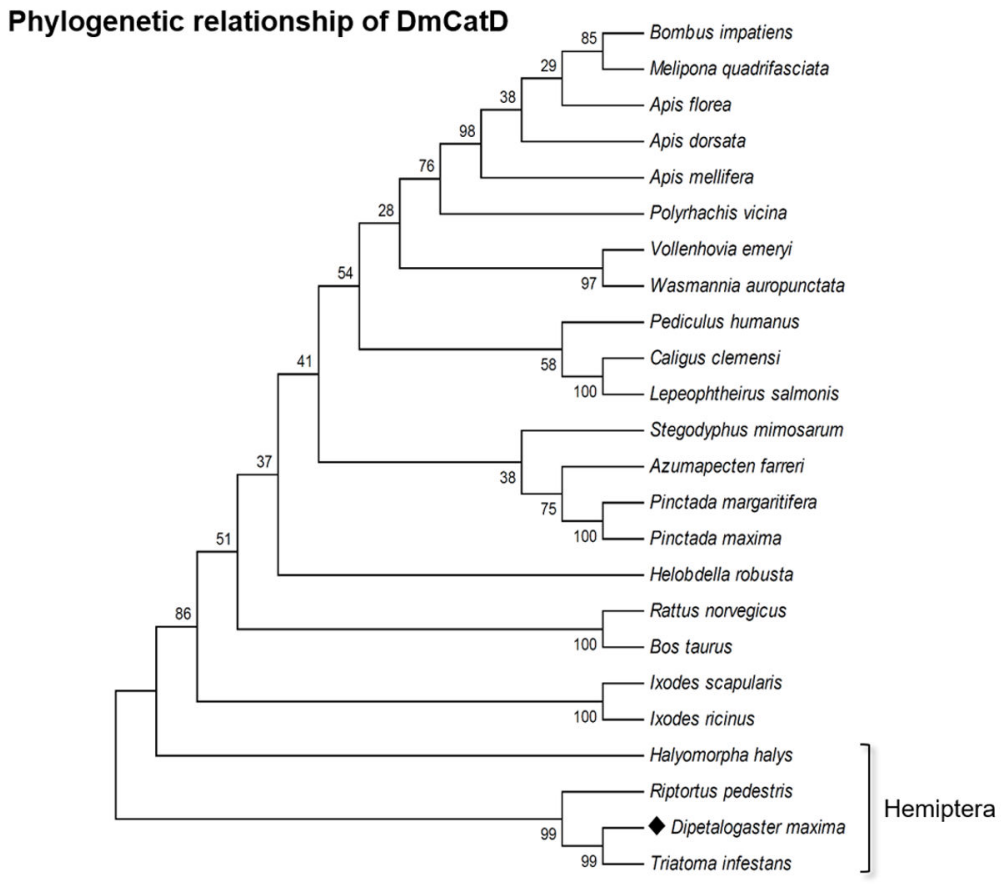

Fig. 4.

Molecular phylogenetic analysis of cathepsin D from selected taxa (bootstrap consensus tree). Cathepsins D from Hemiptera cluster together but not in the pattern expected from species-level phylogeny. The sequences employed for neighbor-joining tree are deposited in GenBank under IDs ABO26561.1 (Ixodes ricinus), ACL13150.1 (Azumapecten farreri), ACO14843.1 (Caligus clemensi), ADD38128.1 (Lepeophtheirus salmonis), ADK47877.1 (Triatoma infestans), AEC03508.1 (Polyrhachis vicina), AEI58896.1 (Pinctada maxima), AFE48185.1 (Pinctada margaritifera), AHE57676.1 (Dipetalogaster maxima), BAN20201.1 (Riptortus pedestris), KFM69649.1 (Stegodyphus mimosarum), KOX72022.1 (Melipona quadrifasciata), NP_599161.2 (Rattus norvegicus), NP_001159993.1 (Bos taurus), XP_002412838.1 (Ixodes scapularis), XP_002427417.1 (Pediculus humanus corporis), XP_003489428.1 (Bombus impatiens), XP_003693293.1 (Apis florea), XP_006607509.1 (Apis dorsata), XP_009027282.1 (Helobdella robusta), XP_011686795.1 (Wasmannia auropunctata), XP_011865870.1 (Vollenhovia emeryi), XP_014270708.1 (Halyomorpha halys), and XP_392857.2 (Apis mellifera). The sequences corresponding to the hemipteran species are indicated by a bracket. 


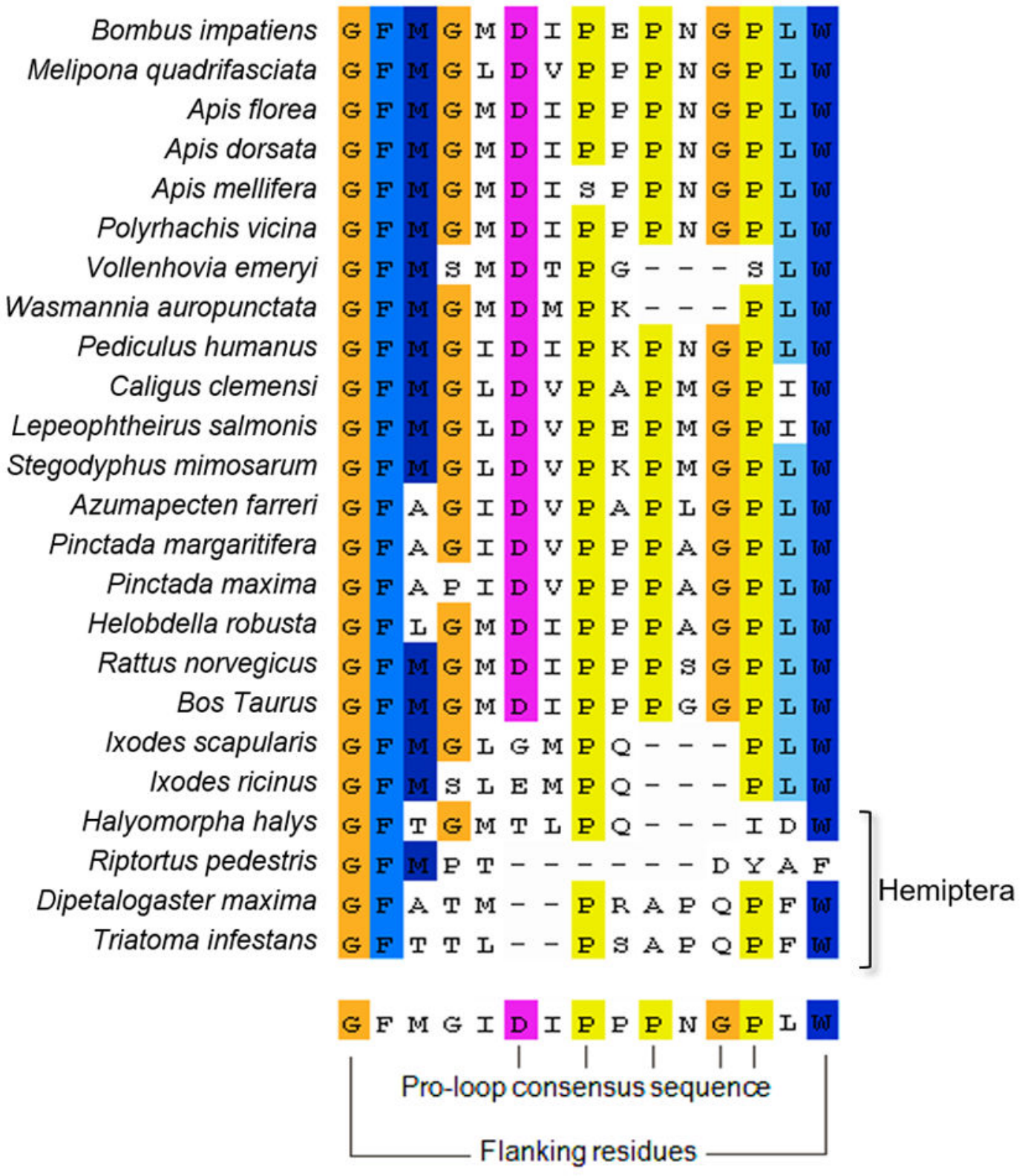

Fig. 5.

Alignment of the proline-loop region. All sequences employed in the phylogenetic inference were aligned by the proline-loop region. The consensus sequence for this motif is shown below the alignment, the conserved amino acids are highlighted using the same color and the sequences corresponding to the hemipteran species are indicated by a bracket. 
(A)

Co-immunoprecipitation of endogenous

lipophorin (Lp) and DmCatD

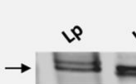

$p^{u^{2}}$

Co-IP

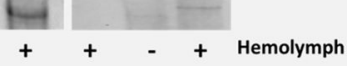

$-$

Rat brain (control)

$+\quad-\quad+\quad-$ Anti-Cat D

- $\quad$ - $\quad$ - $\quad+$ Anti-ATP5b (control)

(B) Co-immunoprecipitation of endogenous

vitellogenin $(\mathrm{Vg})$ and DmCatD

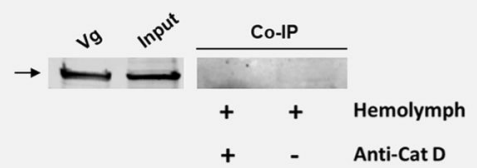

Fig. 6.

(A), Co-immunoprecipitation (Co-IP) of endogenous lipophorin (Lp) and DmCatD. The hemolymph of vitellogenic females or rat brain homogenates (control) were incubated with $0.2 \mu \mathrm{g}$ of anti-cathepsin D or $0.2 \mu \mathrm{g}$ of anti-ATP5b antibodies (control). Samples were transferred to nitrocellulose membranes and then probed with an anti-lipophorin antibody $(1: 1,000)$. Arrow indicates apoLp-II subunit $(\sim 80 \mathrm{kDa})$. Lp line: purified Lp loaded as reference. The presence of endogenous Lp was visualized by loading hemolymph and probing the sample with the anti-lipophorin antibody (Input). (B), Co-immunoprecipitation of endogenous vitellogenin $(\mathrm{Vg})$ and DmCatD. The hemolymph of vitellogenic females was incubated with $0.2 \mu \mathrm{g}$ of anti-cathepsin D antibodies. Samples were transferred to nitrocellulose membranes and then probed with anti-vitellin antibody $(1: 1,000)$. The main subunits of $\mathrm{Vg}(\mathrm{Mr} \sim 170 \mathrm{kDa}$ and $174 \mathrm{kDa})$, visualized as a single immunoreactive band, are shown with an arrow. Vg lane: purified $\mathrm{Vg}$ loaded as a reference. The presence of endogenous $\mathrm{Vg}$ was visualized by loading hemolymph and probing the sample with the antivitellogenin antibody (Input). 


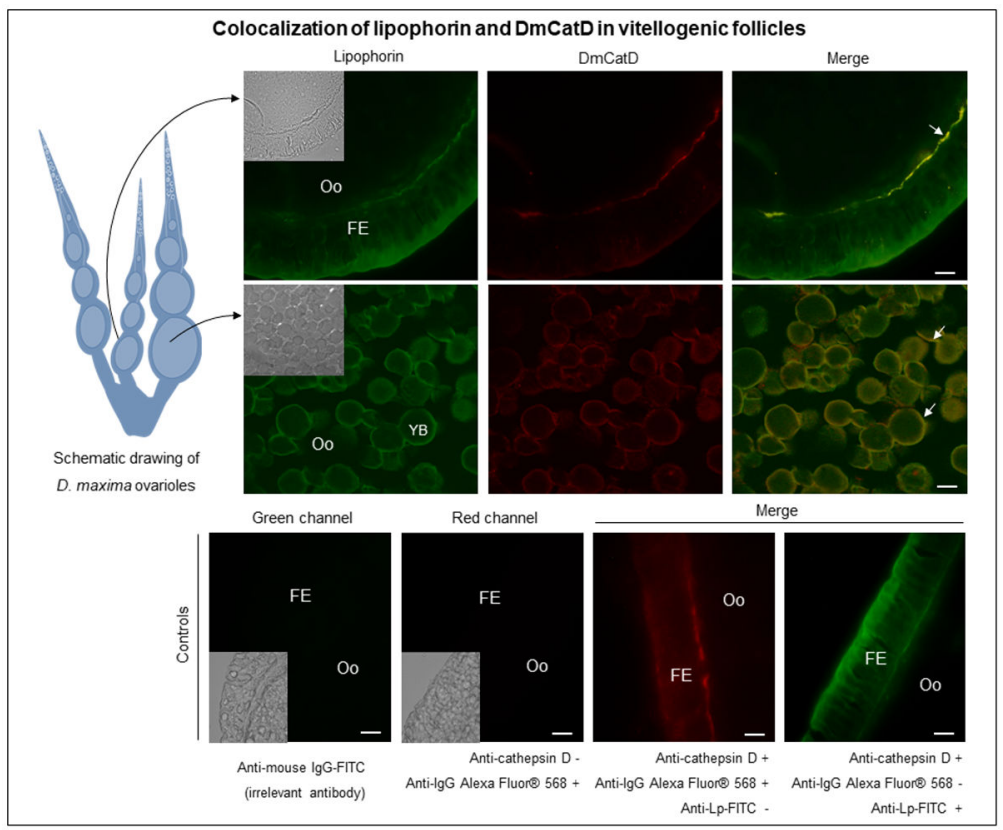

Fig. 7.

Colocalization of lipophorin (Lp) with DmCatD in vitellogenic follicles. Ovaries from females at 4-6 days post-blood feeding were processed for immunofluorescence as stated in Materials and Methods. A schematic drawing of three D. maxima ovarioles is shown to identify the vitellogenic follicles analyzed by immunofluorescence assays. Upper panel: The cathepsin D signal is displayed in red and the Lp signal is in green. In the merged images, the colocalization of Lp with DmCatD is shown by arrows. Insets correspond to DIC images. Similar results were obtained in three separate experiments. Lower panel: immunofluorescence negative controls. The channel displayed (red, green or both in the case of the merged images) is indicated in each figure. Oo, oocyte; FE, follicular epithelial cells; YB, yolk body. Bars: $10 \mu \mathrm{m}$. 


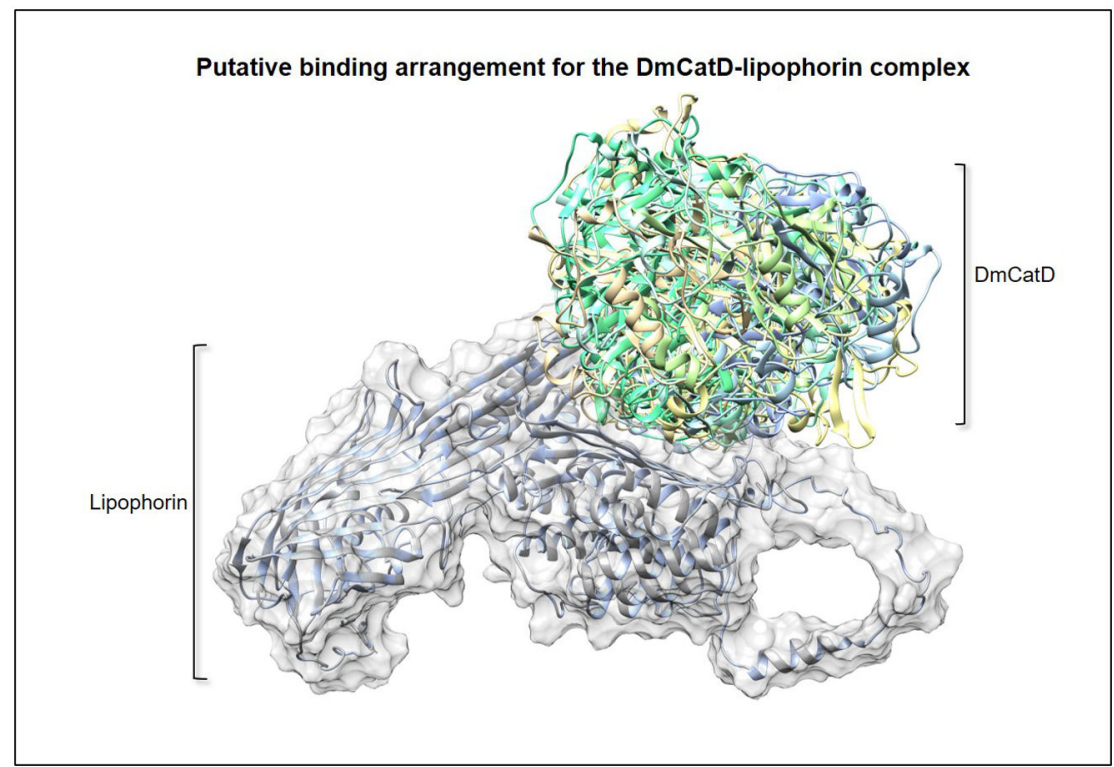

Fig. 8.

Arrangement for putative binding of the complex between chimeric cathepsin D and lipophorin. Ten top-ranked docking solutions are shown as colored cathepsin D monomers bound to lipophorin, depicted in grey cartoon with superposed molecular surface. The solutions are densely concentrated in the same spot of the protein, reinforcing its high probability as a binding site. 


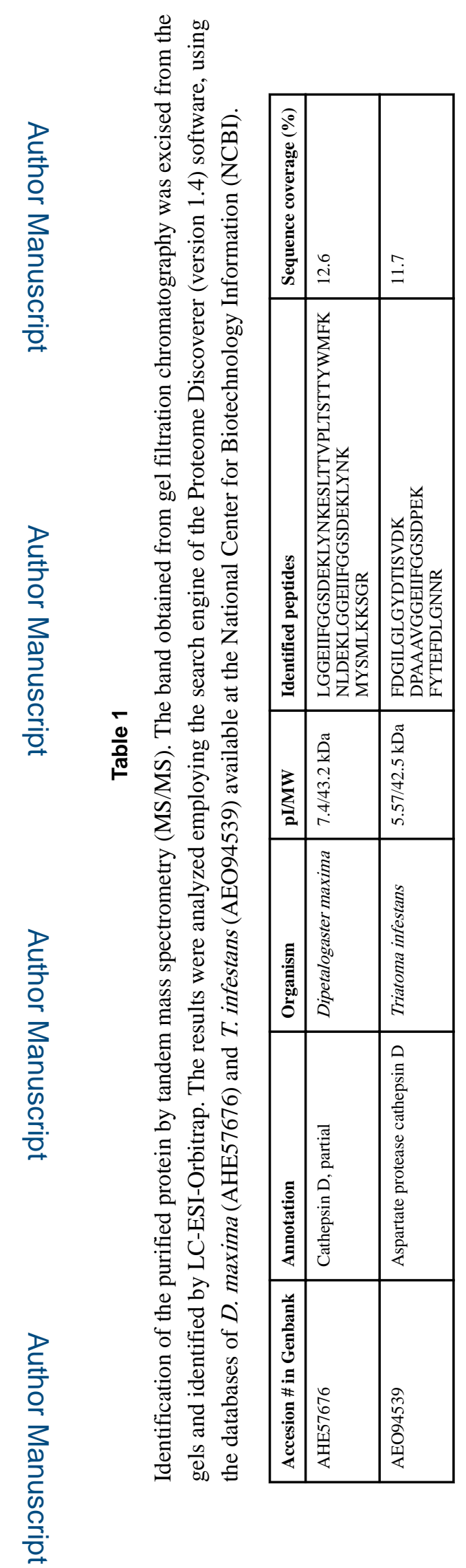

J Insect Physiol. Author manuscript; available in PMC 2019 February 01. 